- Neurological disorders may present in various ways including sensory disturbance, paralysis, altered level of consciousness, fits, speech disturbance, changes in muscle tone or bulk and tremor.

- Facial paralysis may be caused by stroke (upper motor neurone) or Bell's Palsy (lower motor neurone). Surgery in the region of the facial nerve, particularly parotid surgery may also cause dysfunction of the nerve.

- Facial sensory loss (trigeminal nerve) may be caused by extracranial nerve injury. Other causes include multiple sclerosis, stroke and tumours.

- Patients with epilepsy may severely damage the oro-facial tissues in Grand Mal attacks. A good history will alert the practitioner to those who are poorly controlled.

- Some of the drugs used for the treatment of neurological disorders impact on dental disease and its management.

- In patients with severe or complex neurological disorders, consideration should be given to

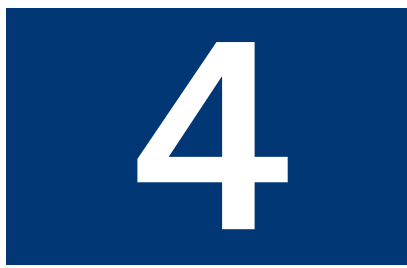
referring such patients for management in a hospital setting.

\title{
General medicine and surgery for dental practitioners Part 4: Neurological disorders
}

\author{
M. Greenwood ${ }^{1}$ and J. G. Meechan ${ }^{2}$
}

\author{
There are a number of neurological conditions that may be encountered in dental practice. \\ It is important that a dental practitioner has a broad knowledge of the main neurological \\ conditions since they may affect the provision of dental treatment.
}

\section{GENERAL MEDICINE AND SURGERY FOR DENTAL PRACTITIONERS:}

1. Cardiovascular system

2. Respiratory system

3. Gastrointestinal system

4. Neurological disorders

5. Liver disease

6. The endocrine system

7. Renal disorders

8. Musculoskeletal system

9. Haematology and patients with bleeding problems

10. The paediatric patient
${ }^{1}$ Lecturer, ${ }^{2}$ Senior Lecturer, Department of Oral and Maxillofacial Surgery, The Dental School, Framlington Place, Newcastle upon Tyne NE2 4BW Correspondence to: M. Greenwood E-mail: beryl.leggatt@ncl.ac.uk

\section{Refereed Paper}

doi:10.1038/sj.bdj.4810275

( ) British Dental Journal 2003; 195:

19-25

\section{RELEVANT POINTS IN THE HISTORY}

The patient may give a history of 'blackouts'. It is important to be precise about what a patient means by this term as this can indicate anything from a loss of consciousness (LOC) to dizziness. When a history of blackouts is given, information obtained from a witness might be useful. The more that is known about the nature of such an event, the better it can be anticipated and effectively managed (or prevented).

The main points in the history are summarised in Table 1.

Syncope may be vaso-vagal in origin (the simple faint) or may occur in response to certain situations such as coughing. A vaso-vagal attack may be precipitated by the fear of dental treatment, heat or a lack of food. It occurs due to a reflex bradycardia and peripheral vasodilation. Onset is not instantaneous and the patient will look pale, often feel sick and notice a 'closing in' of visual fields. It cannot occur when a patient is lying down and placing the patient flat with legs raised is the treatment. Jerking of limbs may occur. In carotid sinus syncope, hypersensitivity of the carotid sinus may cause syncope to occur on turning the head. Unlike vaso-vagal syncope this may happen in the supine position.

Patients may suffer from epilepsy. If this presents as a blackout the most likely type is Grand Mal. A description of the fit is useful as this may enable early recognition. Precipitating factors should be asked about, and enquiry made about altered breathing, cyanosis or tongue biting during a fit. The latter is virtually a diagnostic feature of this type of epilepsy. Medication taken and its efficacy should be assessed in terms of the degree of control achieved. Tonic-clonic or Grand Mal epilepsy is classically preceded by a warning or aura which may comprise an auditory, olfactory or a visual hallucination. A loss of consciousness follows leading to convulsions and subsequent recovery. The patient may be incontinent during a fit. The 'tonic phase' gives way to a 'clonic phase' in which there is repetitive jerky movements, increased salivation and marked bruxism. After a fit of this type, a patient may sleep for up to 12 hours. If the fit continues for more than 5 minutes or continues without a proper end point, 'status epilepticus' is said to be

Table 1 Main points in the history in the dental patient with possible neurological disorders

\footnotetext{
- Blackouts, syncope

- Epilepsy

- Stroke, Transient Ischaemic Attack

- Multiple Sclerosis

- Facial pain

- Parkinson's Disease

- Motor Neurone Disease

- Cranial Nerve problems (especially Bell's Palsy)
} 


\begin{tabular}{ll} 
Table 2 Possible causes of loss of consciousness or 'Blackout' \\
\hline Vaso-Vagal Syncope & Simple faint \\
\hline 'Situational' Syncope & $\begin{array}{l}\text { Cough } \\
\text { Micturition } \\
\text { Carotid sinus hypersensitivity }\end{array}$ \\
\hline Epilepsy & \\
\hline Hypoglycaemia & \\
\hline Transient Ischaemic Attack & $\begin{array}{l}\text { On standing from lying } \\
\text { Orthostatic Hypotension }\end{array}$ \\
\hline reflexes eg elderly patients on \\
tablets to lower blood pressure \\
\hline 'Drop attacks' & $\begin{array}{l}\text { Sudden weakness of legs usually } \\
\text { resolved spontaneously }\end{array}$ \\
\hline Stokes-Adams Attacks & Transient arrhythmia \\
\hline Anxiety & \\
\hline Menieres Disease & Vertigo, tinnitus, hearing loss \\
\hline Choking &
\end{tabular}

present. This is an emergency situation which requires urgent intervention with a benzodiazepine eg intravenous diazepam.

Absence seizures or 'Petit Mal' tend to occur in children who may suddenly arrest speech, attention and movement. So-called 'partial' seizures may be simple or complex. Simple seizures consist of clonic movements of a group of muscles or a limb. Complex seizures may involve hallucinations of hearing, sight or taste.

Febrile convulsions are common in infancy and do not predict progression to later epilepsy. Keeping the child cool with fans, paracetamol and sponging with tepid water are the mainstays of treatment.

Stokes-Adams attacks describe losses of consciousness occurring as a result of cardiac arrhythmias. These may happen with the patient in any position and may occur with no warning except for an awareness of palpitations. Recovery is usually within seconds. Other potential causes of 'blackouts' are shown in Table 2.

The patient may give a history of a stroke (Cerebrovascular Accident - CVA) or a socalled mini-stroke (Transient Ischaemic Attack - TIA). A CVA may be haemorrhagic (subarachnoid, cerebral), thrombotic or embolic in origin. A subarachnoid haemorrhage results from rupture of a Berry aneurysm of the Circle of Willis (which lies at the base of the brain). Subarachnoid haemorrhages tend to affect a younger age group than the other types of CVA and typically patients give a history of a sudden onset of excruciatingly severe headache. The prognosis is poor, but has been improved by surgical and radiological obliteration of the aneurysm. Hypertension and atherosclerosis are contributory factors to other types of CVA. Cerebral thrombosis deprives the brain of part of its blood supply and is the most common type of stroke. Emboli leading to stroke can arise on the wall of an atrium that is fibrillating or from the wall of a heart damaged after a myocardial infarction. Typical results of a CVA are a sudden loss of consciousness, hemiplegia (on the opposite side to the cerebral lesion) and there may be a loss of speech or slurred speech when the CVA affects the left side of the brain. TIA's comprise a sudden onset of focal CNS signs or symptoms due to a temporary occlusion of part of the cerebral circulation. They are frequently associated with partial or complete stenosis of the carotid artery system. The symptoms resolve in less than 24 hours (usually much more quickly). They are harbingers of a CVA and the known patient will usually be taking prophylactic aspirin.

Patients with Multiple Sclerosis have a diverse condition comprising neurological signs and symptoms that are disseminated in both site and time. A viral aetiology has been postulated but the cause is not known. Onset is variable but optic neuritis can lead to visual disturbance or blindness, which may be a presenting feature. Weakness or paralysis of a limb can occur. Nystagmus (jerky, oscillating movement of the eyes - which can also be physiological) may occur, as may ataxia (uncoordinated movements) and dysphagia. Loss of sphincter control leading to urinary incontinence may occur. The diagnosis should be considered in a young patient presenting with trigeminal neuralgia or a facial palsy. Enquiry in such cases should be directed towards other areas to check for neurological signs or symptoms elsewhere.

\section{FACIAL PAIN}

Facial pain is common and may affect up to $50 \%$ of the elderly population. ${ }^{1}$ A paroxysm of excruciating stabbing pain lasting only seconds, in the trigeminal nerve distribution suggests trigeminal neuralgia, ${ }^{2}$ particularly in patients over 50 years of age. In the vast majority of cases it is unilateral and most commonly affects the mandibular and maxillary divisions. Often a 'trigger area' may be identified from the history that is stimulated by washing or shaving for example. Talking may be enough to stimulate the pain. Usually the trigger is easily identified. Carbamazepine or phenytoin are the mainstays of treatment.

Other neurological causes of facial pain include post-herpetic neuralgia and atypical facial pain. In post-herpetic neuralgia the patient complains of a burning pain (often in the ophthalmic division of the trigeminal nerve), which may become chronic. There is no really successful treatment, transcutaneous nerve sectioning and LA infiltration in the painful area has been tried as has carbamazepine. Tricyclic antidepressants have also been used.

When all other causes (including non-neurological causes of facial pain) have been excluded, some patients may still complain of facial pain - usually unilateral. The pain is described as severe, constant and not relieved by analgesics. This type of pain is more common in young females and many are prescribed antidepressants (although not always to curative effect). Atypical facial pain is the term applied to this type of facial pain. 


\section{OTHER DISORDERS}

Parkinson's Disease results from degeneration of the pigmented cells of the substantia nigra leading to dopamine deficiency. The incidence is equal in males and females. The disease may also result from previous head injury or cerebrovascular disease. Clinically the patient may have tremor in the arms and hands (the latter being described as 'pill-rolling'). A so-called 'cogwheel' type of rigidity may be seen on movement. Slow movements (bradykinesia) and restlessness (akathisia) may also be noted. The patient may have an expressionless face and a stooped posture. Impaired autonomic function may lead to a postural drop in blood pressure and hypersalivation resulting in drooling of saliva.

Motor Neurone Disease comprises a group of disorders that affect motor neurones at various levels. There is no sensory loss and this helps differentiation from multiple sclerosis. The aetiology is unknown, but a viral agent is thought possible. Oral hygiene may be difficult in these patients and dysphagia and drooling may occur.

Tumours may arise in various components of the CNS and may be primary or metastatic, the latter being more common in the brain. Benign brain tumours are still a significant problem as they may cause pressure effects and may not be amenable to surgery due to their site. Headaches are characteristically worse in the morning. Tumours from which cerebral metastases arise include lung, breast, GIT and kidney.

Impairment of vision may occur and this may vary from mild disability to complete blindness. Diplopia or double vision may occur after a 'blowout' fracture of the orbital floor ${ }^{3}$ or injury to cranial nerves III, IV and VI. Transient visual disturbance may occur secondary to local anaesthetic that has tracked to the inferior orbital fissure.

Myasthenia Gravis (MG) is an antibodymediated autoimmune disease with a deficiency of functioning muscle acetylcholine receptors that leads to muscle weakness. The disorder more commonly affects young women. The muscle weakness is progressive and develops rapidly. Some cases are associated with the Eaton-Lambert Syndrome that may occur in some patients with lung or other cancers. In the Eaton-Lambert syndrome, however, the muscles get stronger rather than weaker with activity.

A facial palsy may have a known cause or be idiopathic (Table 3). If the cause is not known the name Bell's Palsy (Fig. 1) is applied. Other causes must be excluded before this term is used. In Bell's Palsy the onset is rapid, unilateral and there may be an ache beneath the ear. The weakness worsens over one to two days. If presentation is early, most clinicians give prednisolone for 5 days, the aim being to reduce neuronal oedema. An eye patch is of value to protect the cornea. The paralysis is of a lower motor neurone type in which all the facial muscles are affected on that side. In an upper motor neurone lesion eg stroke, the forehead is spared since this region is bilaterally represented in the cortex.

\begin{tabular}{ll}
$\begin{array}{l}\text { Table } 3 \text { Some possible causes of facial weakness } \\
\text { (mostly unilateral) }\end{array}$ \\
\hline Idiopathic & Bell's Palsy \\
Melkersson-Rosenthal Syndrome \\
\hline Infection & Ear infections \\
& TB \\
& Ramsay Hunt Syndrome \\
& Glandular Fever \\
& AlDS \\
\hline Trauma & Facial lacerations/bruising \\
& in the region of the facial nerve \\
& Penetrating parotid injuries \\
& Post-parotid surgery \\
\hline Neoplastic & Primary or secondary cancers \\
& Neuroma of facial nerve \\
& Acoustic neuroma \\
\hline Metabolic & Diabetes Mellitus \\
& Pregnancy \\
& Sarcoidosis \\
Guillain-Barre Syndrome
\end{tabular}

Looking for 'forehead sparing' is thus a way of differentiating between upper and lower motor neurone causes of facial weakness.

Bilateral facial paralysis is rare. It may be seen in sarcoidosis or the Guillain Barre Syndrome (idiopathic polyneuritis) or posterior cranial fossa tumours. The rare Melkersson-Rosenthal Syndrome is a condition comprising tongue fissuring, unilateral facial palsy and facial swelling. The lesions are histologically similar to those of Crohn's Disease.

A summary of other cranial nerve lesions is given in Table 4 . Nerves may be affected by a systemic cause eg diabetes mellitus, MS or there may be a local cause. Multiple cranial nerve palsies may occur in Bulbar Palsy that comprises palsy of the tongue, muscles of chewing/swallowing and facial muscles due to loss of function of motor nuclei in the brainstem. The onset may be acute eg in infection such as polio, or may be chronic eg in tumours of the posterior cranial fossa.

Tics or involuntary facial movements may occasionally be seen in patients. These may be habitual, particularly in children or may be drug induced or they may have a more organic cause. Drug induced dyskinesias are common in the elderly on long-term phenothiazine (anti-psychotic) medication which is usually reversible on stopping the drug. Intracranial nerve compression may result in blepharospasm (contraction of both eyelids). Hemifacial muscle spasm may occur and suggests a lesion eg of the cerebellopontine angle compressing the facial nerve. Whenever a facial tic is found, consideration should be given to referral for investigation since an underlying cause may often be treated.

In the Ramsay-Hunt Syndrome, a profound facial paralysis is accompanied by vesicles in the pharynx on the same side and in the external auditory meatus. It is thought that the geniculate ganglion of the facial nerve is infected with herpes zoster.

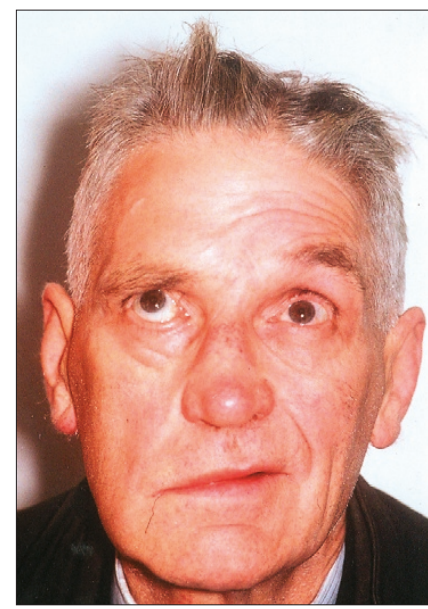

Fig. 1 A patient with Bell's Palsy. The ipsilateral forehead is affected also indicating a lower motor neurone lesion. Bell's sign (see text) is also demonstrated 


\begin{tabular}{|c|c|c|c|}
\hline \multicolumn{2}{|c|}{ Cranial nerve } & \multirow{2}{*}{$\begin{array}{l}\text { Possible problem } \\
\text { Trauma, Tumour }\end{array}$} & \multirow{2}{*}{$\begin{array}{l}\text { Sign } \\
\text { Decreased ability to smell }\end{array}$} \\
\hline | & Olfactory & & \\
\hline$\|$ & Optic & $\begin{array}{l}\text { Trauma, Tumour } \\
\text { MS, Stroke }\end{array}$ & $\begin{array}{l}\text { Blindness, visual field } \\
\text { defect }\end{array}$ \\
\hline III & Oculomotor & $\begin{array}{l}\text { Diabetes, increased intra- } \\
\text { cranial pressure }\end{array}$ & $\begin{array}{l}\text { Dilated pupil } \\
\text { Ptosis }\end{array}$ \\
\hline IV & Trochlear & Trauma & Diplopia \\
\hline \multirow[t]{3}{*}{ V } & Trigeminal & $\begin{array}{l}\text { Sensory - idiopathic, } \\
\text { trauma, IDN/Lingual } \\
\text { nerve damage }\end{array}$ & $\begin{array}{l}\text { None, sensory } \\
\text { deficit on testing }\end{array}$ \\
\hline & & Motor - Bulbar palsy & Signs in $I X, X, X I, X I I$ \\
\hline & & Acoustic Neuroma & $\begin{array}{l}\text { May be decreased facial sensation. } \\
\text { Affects VIII also }\end{array}$ \\
\hline $\mathrm{VI}$ & Abducens & MS, some strokes & $\begin{array}{l}\text { Inability of eye to look } \\
\text { laterally } \\
\text { Eye deviated towards nose }\end{array}$ \\
\hline \multirow[t]{2}{*}{ VII } & Facial & $\begin{array}{l}\text { LMN - Lower motor neurone } \\
\text { Bell's Palsy, Skull fracture } \\
\text { Parotid tumour }\end{array}$ & $\begin{array}{l}\text { Total } \\
\text { Facial weakness }\end{array}$ \\
\hline & & $\begin{array}{l}\text { UMN - Upper motor neurone } \\
\text { Stroke, Tumour }\end{array}$ & $\begin{array}{l}\text { Forehead sparing } \\
\text { weakness }\end{array}$ \\
\hline VIII & $\begin{array}{l}\text { Vestibulo- } \\
\text { cochlear }\end{array}$ & $\begin{array}{l}\text { Excess noise; } \\
\text { Paget's, acoustic neuroma }\end{array}$ & Deafness \\
\hline IX & $\begin{array}{l}\text { Glosso- } \\
\text { pharyngeal }\end{array}$ & Trauma, Tumour & Impaired gag reflex \\
\hline $\bar{x}$ & Vagus & Trauma, brainstem lesions & $\begin{array}{l}\text { Impaired gag reflex } \\
\text { Soft palate moves to } \\
\text { 'good' side on saying } \\
\text { 'aah'. }\end{array}$ \\
\hline$\overline{X I}$ & Accessory & Polio, stroke & $\begin{array}{l}\text { Weakness turning head } \\
\text { away from affected side } \\
\text { (sternocleidomastoid). } \\
\text { Weakness shrugging } \\
\text { shoulders (trapezius) }\end{array}$ \\
\hline XII & Hypoglossal & $\begin{array}{l}\text { Trauma } \\
\text { Brainstem lesions }\end{array}$ & $\begin{array}{l}\text { Tongue deviated to } \\
\text { affected side on } \\
\text { protrusion }\end{array}$ \\
\hline
\end{tabular}

Infections affecting the nervous system may be bacterial or viral in origin. The possibility of bacterial meningitis should be borne in mind with maxillofacial injuries involving the middle third of the face. Prompt treatment with antimicrobials (prophylactically in trauma cases) should be undertaken. The viral type of meningitis is usually mild and self-limiting. The patient with meningitis has a severe headache, feels sick or actively vomits and is often drowsy. The painful, stiff neck and aversion to light are well known. In meningitis caused by the bacterium Neisseria meningitidis, a purpuric rash may be seen on the skin and can progress to adrenocortical failure as a result of bleeding into the adrenal cortex.

Herpetic encephalitis is rare, but should be treated promptly with aciclovir. In HIV associated neurological disease, a wide variety of infections and tumours are seen, for example lymphomas. Neurological effects of such lesions vary from fits to limb weakness.

Brain abscess is a condition that may be secondary to oral sepsis ${ }^{4}$ or infection elsewhere eg the middle ear or paranasal sinuses. A patient with congenital heart disease is also at increased risk. Such abscesses can be a complication of infective endocarditis which should be specifically asked about. Signs and symptoms resulting from a brain abscess depend on its location and $\mathrm{CT}$ and MRI scanning are useful in localisation and diagnosis. Urgent surgical drainage is required.

Cerebral Palsy is primarily a disorder of motor function secondary to cerebral damage, most frequently associated with birth injury or hypoxia. It is the most common cause of a congenital physical handicap, the patterns of which are variable. There are three main subtypes spastic, ataxic and athetoid varieties. In the spastic type the muscles are contracted and there may be associated epilepsy. In the ataxic type, a cerebellar lesion is responsible for a disturbance of balance. Writhing movements characterise the athetoid type of cerebral palsy.

In spina bifida, the vertebral arches fail to fuse, possibly due to a deficiency of folic acid during foetal development. The condition may lead to significant physical handicap such as an inability to walk, epilepsy or learning difficulties. There may be an association with hydrocephalus which often requires decompression using a shunt. This is discussed further in the paediatric paper (Part 10 of this series).

Patients with syringomyelia have a condition in which cavitation of the central spinal cord occurs leading to a loss in pain and temperature sensibility. Syringobulbia is the term used if the brain stem is affected - facial sensory loss may occur, as may tongue weakness.

An acoustic neuroma is a benign tumour occurring at the cerebello-pontine angle on the vestibular part of the vestibulocochlear nerve. Cranial nerves V, VII, IX and X may also be involved leading to tinnitus, deafness and vertigo. Facial twitching, weakness or paraesthesias may occur. Other causes of facial sensory loss (innervated by the trigeminal nerve except over the angle of the mandible which is innervated by cervical nerves) are given in Table 5.

Other neurological disorders that may be encountered include Huntington's Chorea, which is an autosomal dominant disorder where there is progressive dementia with marked involuntary movements. The signs do not begin to

\begin{tabular}{|c|c|}
\hline \multicolumn{2}{|l|}{ Intracranial } \\
\hline - Neoplasm & - Cerebral Tumour \\
\hline \multicolumn{2}{|c|}{$\begin{array}{l}\text { Granulomatous conditions eg sarcoid TB } \\
\text { Connective tissue disorders }\end{array}$} \\
\hline \multicolumn{2}{|c|}{$\begin{array}{l}\text { Other : Paget's Disease (nerve compression) } \\
\text { Trigeminal Neuropathy } \\
\text { Cerebrovascular Disease }\end{array}$} \\
\hline \multicolumn{2}{|l|}{ Extracranial } \\
\hline - Neoplasm & - Cancer, Metastatic Cancers \\
\hline - Inflammatory & $\begin{array}{l}\text { - Osteomyelitis } \\
\text { - Pressure from adjacent lesions }\end{array}$ \\
\hline - Trauma & $\begin{array}{l}\text { - Maxillary/mandibular fractures } \\
\text { latrogenic eg removal of mandibular } \\
\text { third molars }\end{array}$ \\
\hline
\end{tabular}


appear until middle age. Friedreich's Ataxia is an autosomal recessive or sometimes sex-linked cord degeneration of unknown cause. Severe ataxia and deformity occur and there may be associated cardiac disease with arrhythmias.

\section{GENERAL EXAMINATION}

The patient's gait may give an immediate clue to an underlying neurological condition, for example the shuffling gait of Parkinsonism. A spastic gait is demonstrated by stiff limbs that are often swung around in a circular motion as the forward movement proceeds.

The patient with a neurological condition may appear confused. This may be for several reasons and a summary of potential causes is given in Table 6. Raised intracranial pressure can occur following trauma to the head for example in a patient attending with an injury associated with dental trauma. Such patients will complain of headache and may be restless or vomiting. The classical sign of increased blood pressure and decreased pulse rate occur and there is dilation of the pupil on the same side as the lesion. Patients with a head injury can be assessed according to the Glasgow Coma Scale

\begin{tabular}{l}
$\begin{array}{l}\text { Table } 6 \text { Possible causes of confusion that may be } \\
\text { encountered in a dental patient }\end{array}$ \\
\hline Hypoxia - Ensure clear airway, care with sedatives \\
\hline Epilepsy \\
\hline Infection - Significant oro-facial infection, pneumonia, \\
meningitis \\
\hline Metabolic - Hypoglycaemia \\
\hline Drug/Alcohol withdrawal \\
\hline Vascular - Stroke, Ml \\
\hline Raised intracranial pressure \\
\hline Nutritional - Deficiency of various B vitamins
\end{tabular}

(GCS) which involves three scored categories of assessment - eye opening, muscle responses and responses to vocal stimuli.

Horner's Syndrome comprises the four signs of a constricted pupil, ptosis (drooping of the upper eyelid), loss of sweating on the ipsilateral face and enophthalmos. It is caused by interference with the cervical sympathetic chain eg after a radical neck dissection, trauma to the neck or tumour.

Patients with cerebral palsy have an increased incidence of dental malocclusion and abnormal movement of the oral and facial musculature that may cause difficulty in dental treatment provision.

The Sturge-Weber syndrome (Fig. 2) describes an association between a facial port wine stain (haemangioma) with focal fits on the contralateral side. Exophthalmos and spasticity may also be evident. The fits are caused by a capillary haemangioma in the brain.

\section{CONSIDERATION OF THE CRANIAL NERVES}

A systematic approach is needed for examining the Cranial Nerves. One approach is to consider

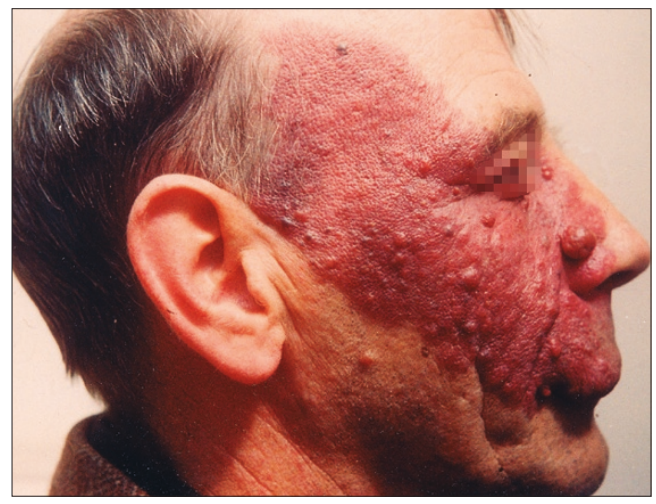

Fig. 2 A patient with Sturge Weber Syndrome

the cranial nerves in the following groups: ie nerve(s) subserving the sense of smell, eyes, face, mouth, neck and ears. A summary of disorders affecting the cranial nerves and resulting signs is given in Table 4.

Any changes in the sense of smell may reflect a problem with the olfactory nerve. Colds and sinusitis may be the cause but trauma involving the cribriform plate can also cause the nerve to have impaired function. Some operations on the nose may cause injury to the olfactory nerves.

Visual acuity may be roughly assessed by asking the patient to read a printed page. Defects of the optic nerve may also affect the field of vision. A lesion of the cranial nerve III leads to complete or partial ptosis (drooping of the upper eyelid). The external ocular muscles are controlled by the action of cranial nerves III, IV and VI. Disruption of the third nerve (which supplies all of the extrinsic eye muscles apart from superior oblique and lateral rectus) causes a paralysis of internal, upward and downward movement of the eye leading to double vision. The eye points downwards and outwards except when looking to the affected side. A fixed dilated pupil may also be seen. Disruption of IV, the trochlear nerve supplying superior oblique, prevents the eye moving downwards and medially. The double vision is worse on looking down. Disruption of VI (abducens supplying lateral rectus) causes an inability to abduct the eye (look to the ipsilateral side). There is deviation of the eye towards the nose and double vision.

The muscles of facial expression are innervated by the VII cranial nerve (facial nerve). As mentioned previously, upper motor neurone lesions affecting the facial nerve eg after a stroke may be differentiated from lower motor neurone causes eg Bell's Palsy since the latter causes the whole side of the face to be weakened whereas the forehead is spared in an upper motor neurone lesion due to bilateral representation at the level of the cerebral cortex. The ipsilateral eye moves upwards on attempted closure of the eyes in Bell's Palsy this is known as Bell's Sign (Fig. 1).

In terms of facial sensation the sensory division of the trigeminal ( $\mathrm{V}$ cranial) nerve subserves this over most of the face. The ophthalmic, maxillary and mandibular divisions may be compared by testing skin sensation on
Eye signs

Problems with cranial nerves II, III, IV and VI affect the eyes 


\section{Cranial nerves \\ Defects in cranial nerves $\mathrm{V}, \mathrm{IX}, \mathrm{X}$ and $\mathrm{XII}$ have intra-oral manifestations}

either side with a wisp of cotton wool. The corneal (blink) reflex is often the first clinical deficit to be seen in trigeminal nerve lesions.

The mouth can demonstrate signs of cranial nerve problems in the case of cranial nerves $\mathrm{V}$ (motor division), IX, X and XII. If the masseter muscles are palpated whilst asking the patient to clench the teeth and the motor division of $\mathrm{V}$ is inactive, the masseter on that side will not contract properly. With a unilateral lesion, the mandible deviates to the weak side on opening the mouth ( $\mathrm{V}$ being motor to the pterygoid muscles).

Asking the patient to say 'aah' will allow an appraisal of IX and X nerves. The ninth (glossopharyngeal) nerve is mainly sensory for the pharynx and palate and the tenth (vagus) mainly motor. With a unilateral lesion of the vagus, the soft palate is pulled away from the weaker side. Lesions of both nerves lead to an impaired gag reflex. The twelfth (hypoglossal) nerve may be tested by asking the patient to protrude the tongue. The tongue deviates to the weaker side.

To test the accessory (eleventh) cranial nerve the patient should be asked to put their chin towards the left or right shoulder against resistance by the examiner. The sternocleidomastoid muscle (supplied by XI) does not function when $\mathrm{XI}$ is affected.

The eighth (vestibulocochlear) nerve has two components - the vestibular (appreciation of position and movements of the head) and the cochlear (responsible for hearing). Lesions of the nerve may cause hearing loss, vertigo or ringing in the ears (tinnitus). Special tests are needed to test the balance and positional functions of the nerve.

\section{GENERAL AND LOCAL ANAESTHESIA, SEDATION AND MANAGEMENT CONSIDERATIONS IN THE DENTAL PATIENT WITH NEUROLOGICAL DISEASE}

Patients prone to syncope should be treated with regard to avoidance of known precipitating factors as far as possible. Treatment in the supine position has obvious advantages.

Epileptic patients referred for GA should not be given methohexitone or enflurane since these are epileptogenic. It is important to ensure that an epileptic patient has taken their normal medication on the day of the procedure. Intravenous sedation is useful in managing epileptic patients. The benzodiazepines have anticonvulsant properties and anxiolysis should decrease the chances of a fit. When treating epileptic patients with sedation supplemental oxygen should be provided via a nasal cannula. ${ }^{5}$ The use of the benzodiazepine reversal agent flumazenil should be avoided in patients with epilepsy as this drug can precipitate convulsions.

Patients who have had a CVA should have treatment only when their condition has been optimised if possible. There may be a loss of reflexes such as swallowing or the gag reflex, which has implications for the safe provision of treatment under LA, with or without sedation. Ability to protect the airway is also relevant for the provision of GA since all these modes of treatment jeopardise the airway to some extent. Stroke patients may be taking anticoagulants, or may be hypertensive.

In both multiple sclerosis and motor neurone disease, the degree of compliance achievable for treatment is likely to be impaired. It is best to use LA alone if possible. Limited mobility and/or associated psychological disorders may cause difficulties with treatment. Patients are better treated sitting so that respiration is assisted as much as possible since it may be impaired. Patients with MS may be taking corticosteroids, particularly early in the disease. Care of the airway may be made more difficult due to muscular incoordination.

Patients with Parkinson's disease suffer from excess salivation, which can cause difficulties with visibility leading to problems not only providing the treatment itself but also for the safe provision of an anaesthetic. Anti-muscarinic drugs will reduce the salivation and degree of tremor. The autonomic insufficiency often found in these patients makes them liable to postural hypotension and poor candidates for general anaesthesia.

In myasthenia gravis, local anaesthesia is the option of choice. Doses should be kept to a minimum. Muscle fatigue appears to increase during the day and therefore treatment is best carried out early. Intravenous sedation should not be given in a dental practice setting ${ }^{6}$ since respiratory impairment may be worsened. A small oral dose of a benzodiazepine is acceptable if the patient is very anxious about their treatment. For similar reasons general anaesthetics are also not advised if possible. In addition, some of the agents used with GA eg the muscle relaxant suxamethonium or opioids eg fentanyl may have their effects potentiated in these patients.

Patients with visual problems may be permanently disabled or the disorder may be transient. It is important that a 'tell-do' approach is used for these patients to minimise anxiety. It is worth bearing in mind in these patients that other senses eg hearing may be heightened. Diplopia can be a transient complication of LA as mentioned earlier. Other cranial nerve lesions of relevance include those affecting cranial nerves IX and $\mathrm{X}$ since the gag reflex may be impaired leading to potential airway compromise, particularly if sedation or GA are being used.

Patients with cerebral palsy may not be able to tolerate treatment under LA, and GA may be the only way of achieving it. In the athetoid type, potential epilepsy should be borne in mind. Anxiety will often worsen the effects of the cerebral palsy and therefore premedication e.g. with diazepam is often wise. Patients with spina bifida have increased incidence of latex allergy. Such patients may be prone to postural hypotension and are therefore best treated sitting up. 
Epilepsy and renal anomalies may also be associated. In Friedreich's Ataxia, possible arrhythmias should be remembered.

\section{EFFECTS OF DRUGS USED IN NEUROLOGICAL DISORDERS ON ORO-DENTAL STRUCTURES}

Drugs used to treat neurological conditions can produce unwanted effects in and around the mouth. Anticonvulsants have a number of unwanted effects of interest to dentists. Gingival overgrowth is a recognised side effect of phenytoin ${ }^{7}$ but may also occur with sodium valproate and ethosuximide. In addition, phenytoin causes taste disturbance and may produce StevensJohnson syndrome. This drug may also affect the teeth. It has been implicated in producing hypercementosis and shortening of the roots. ${ }^{8}$ Sodium valproate may produce parotid gland enlargement. Lamotrigine may cause dry mouth and Stevens-Johnson syndrome. Ethosuximide may produce Stevens-Johnson syndrome and gingival bleeding. Carbamazepine may produce xerostomia, glossitis and oral ulceration. Stevens-Johnson syndrome is the name given to a severe form of erythema multiforme. The latter predominantly affects young males and is characterized by mucosal lesions with or without skin lesions. The typical skin lesion is described as looking like a target as it consists of concentric erythematous rings. The rashes may appear differently however, hence the name 'multiforme'. The aetiology of erythema multiforme is not known but is thought to be a disorder of immune complexes. The antigens can be as diverse as microorganisms or drugs.

The anti-muscarinic anti-Parkinsonian drugs such as orphenadrine and benzhexol can produce dry mouth, which may increase caries incidence. In addition, the dopaminergic drugs such as levodopa and co-careldopa may produce taste disturbances.

\section{SUMMARY}

As with disorders of other systems, neurological diseases impact on dental treatment provision, both in terms of the treatment itself and the provision of safe methods of anaesthesia/analgesia to facilitate it.

1. Madland G, Newton-John T, Feinmann C. Chronic idiopathic Orofacial pain: I: What is the evidence base? Br Dent J2001; 191: 22-24.

2. Nurmikko T J, Eldridge PR. Trigeminal neuralgia pathophysiology, diagnosis and current treatment. Br J Anaesth 2001; 87: 117-132.

3. Courtney D J, Thomas S, Whitfield P H. Isolated orbital blowout fractures: survey and review. Br J Oral Maxillofac Surg 2000: 38: 496-502.

4. Corson M A, Postlethwaite K R, Seymour R A. Are dental infections a cause of brain abscess? Case report and review of the literature. Oral Diseases 2001, 7: 61-65.

5. Meechan J G, Robb N D, Seymour R A. Pain and Anxiety Control for the Conscious Dental Patient. pp287-290. Oxford: Oxford University Press, 1998.

6. Malamed S F. Sedation: a Guide to Patient Management. 3rd ed. p595 - 596. St Louis: Mosby, 1995.

7. Hassell T M. Epilepsy and the Oral Manifestations of Phenytoin Therapy. Basle: Kruger, 1981.

8. Seymour R A, Meechan J G, Walton J G. Adverse Drug Reactions in Dentistry. 2nd ed. p 121. Oxford: Oxford University Press, 1996 